\title{
COMPARISON THEOREMS FOR SECOND ORDER DIFFERENTIAL SYSTEMS
}

\author{
W. J. KIM
}

\begin{abstract}
Comparison theorems are proved for second order linear differential systems of the form $\left(R_{i} v^{\prime}\right)^{\prime}+P_{i} v=0$, where $R_{i}$ and $P_{i}$ are continuous $n \times n$ matrices and $R_{i}$ is invertible, $i=1,2$.
\end{abstract}

Let $R$ and $P$ be $n \times n$ matrices with real elements which are continuous and let $R$ be invertible on an $x$-interval $[a, \omega)$. We shall consider the second-order vector differential equation

$$
\left(R(x) y^{\prime}\right)^{\prime}+P(x) y=0 .
$$

If (E) has a nontrivial solution $v$ satisfying $v(b)=v^{\prime}(c)=0\left[v^{\prime}(b)=v(c)=0\right]$ for some $b$ and $c, a \leqslant b<c<\omega$, we define $\eta(b)[\phi(b)]$ to be the infimum of $\xi$, $b \leqslant \xi<\omega$, such that there exists a nontrivial solution $u$ of (E) satisfying $u(b)=$ $u^{\prime}(\xi)=0\left[u^{\prime}(b)=u(\xi)=0\right]$. Otherwise, we put $\eta(b)=\omega[\phi(b)=\omega]$. If $\eta(b)<\omega$ $[\phi(b)<\omega]$, then (E) has a nontrivial solution $y$ such that $y(b)=y^{\prime}(\eta(b))=0$ $\left[y^{\prime}(b)=y(\phi(b))=0\right] . \phi(b)$ is called the right-hand focal point of $b$. In recent years some authors have referred to $\eta(b)$ as a focal point of $b$; however, this appears to be inconsistent with the long-term usage of "focal" [13]. In Picone's terminology, $\eta(b)$ is a right-hand pseudoconjugate of $b$ and $\phi(b)$ is a right-hand hemiconjugate to $b$. We shall henceforth call $\eta(b)$ the right-hand pseudoconjugate of $b$.

Morse [11] was the first to obtain generalizations of the classical Sturm separation and comparison theorems for the second-order vector differential equations

$$
\left(R_{i}(x) y^{\prime}\right)^{\prime}+P_{i}(x) y=0, \quad i=1,2,
$$

where $R_{i}$ and $P_{i}$ are $n \times n$ matrices with continuous and real elements and $R_{i}$ is invertible on $[a, \omega), i=1,2$. Other comparison results of a different nature have been recently proved by Ahmad and Lazer [1-3] for the case $R_{i}=I$, and also by others $[6,10,14,15]$ under various assumptions on $R_{i}$ and $P_{i}$. In [15] Tomastik also considered comparison theorems for the right-hand and left-hand focal points. It is to be noted that in most of these studies the case $P_{1} \equiv P_{2}$ is specifically excluded.

Received by the editors May 11, 1984 and, in revised form, October 18, 1984.

1980 Mathematics Subject Classification. Primary 34C10.

Key words and phrases. Comparison theorems, second order linear differential systems, focal and pseudoconjugate points. 
Let $\eta_{i}(b)\left[\phi_{i}(b)\right]$ be the right-hand pseudoconjugate [the right-hand focal point] of $b$ for $\left(E_{i}\right), i=1,2$. The purpose of this paper is to present theorems comparing $\eta_{1}(b)\left[\phi_{1}(b)\right]$ and $\eta_{2}(c)\left[\phi_{2}(c)\right]$, where $b$ and $c$ are not necessarily equal. For the special case $R_{1} \equiv R_{2}, P_{1} \equiv P_{2}$, these results become "separation theorems," from which we can further deduce that $\eta_{i}(x)\left[\phi_{i}(x)\right]$ is a nondecreasing function of $x$.

The Riccati equation technique $[5,8,9,12]$ adapted to the second-order system (E) is used to establish the main theorems.

THEOREM 1. Let $b$ be a point on the interval $[a, \omega)$. Every nontrivial vector solution $y$ of (E) with $y(b)=0$ has the property that $y^{\prime}(x) \neq 0, b \leqslant x<\omega$, if and only if the matrix Riccati system

$$
S^{\prime}=R^{-1}+S P S, \quad S(b)=0,
$$

has a solution on $[b, \omega)$.

Proof. Let $Y$ be the solution of the matrix system

$$
\left(R(x) Y^{\prime}\right)^{\prime}+P(x) Y=0, \quad Y(b)=0, \quad Y^{\prime}(b)=I .
$$

To prove the necessity, let $\alpha$ be an arbitrary nonzero constant vector. Then $y(x) \equiv Y(x) \alpha$ is a nontrivial solution of (E) with $y(b)=0$. Since $y^{\prime}(x)=Y^{\prime}(x) \alpha$ $\neq 0, b \leqslant x<\omega$, we see that the determinant of $Y^{\prime}(x)$ does not vanish on $[b, \omega)$. Thus, $Y^{\prime}$ is invertible on $[b, \omega)$. Since $R$ is also invertible on $[b, \omega)$, so is $R Y^{\prime}$. Consequently, $S \equiv Y\left(R Y^{\prime}\right)^{-1}$ is defined and continuously differentiable on $[b, \omega)$ and $S(b)=0$. Differentiating $S$, we obtain $S^{\prime}=R^{-1}+S P S$, which proves that $S$ is a solution of (MR) on $[b, \omega)$.

For $n \times n$ matrices $A=\left(a_{i j}\right)$ and $B=\left(b_{i j}\right)$, we write $A \geqslant B$ if $a_{i j} \geqslant b_{i j}, i$, $j=1, \ldots, n$, and we define

$$
\int_{b}^{x} A(t) d t=\left(\int_{b}^{x} a_{i j}(t) d t\right)
$$

In order to prove the sufficiency, we require the following lemma.

LeMma 1. The matrix Riccati equation (MR) has a unique solution $S$ on $J=$ $[b, \eta(b))$. The solution $S$ is continuously differentiable and nontrivial; furthermore, it is nonnegative on $J$ if

$$
R^{-1}(x) \geqslant 0, \quad P(x) \geqslant 0, \quad b \leqslant x<\eta(b) .
$$

Proof. If $R^{-1}=\left(t_{i j}\right), P=\left(p_{i j}\right)$, and $S=\left(s_{i j}\right)$, the system (MR) is equivalent to the system of $n^{2}$ first-order equations

$$
s_{i j}^{\prime}=t_{i j}+\sum_{k=1}^{n} s_{i k} \sum_{l=1}^{n} p_{k l} s_{l j}, \quad s_{i j}(b)=0,
$$

$i, j=1,2, \ldots, n$. Evidently, the above system may be cast into a vector equation of the form

$$
s^{\prime}=f(x, s), \quad s(b)=0,
$$


where $s$ and $f$ are $n^{2}$-dimensional vectors. The vector-valued function $f$ is continuous on $D=\{(x, s): x \in J,|s|<\infty\}$; indeed, it is continuously differentiable on $D$ as a function of $s$. Therefore, $f(x, s)$ satisfies a Lipschitz condition with respect to $s$ on any compact and convex subset of $D$ (see, e.g., [4, p. 142]) and there exists a unique solution $s \in C^{\prime}$ of (2) on some interval [b,c], $b<c<\eta(b)$ [7, p. 10]. Hence, the matrix Riccati system (MR) has a unique solution $S$, continuously differentiable on the interval $[b, c]$. Let $c_{1}=\sup \{c:(\mathrm{MR})$ has a unique solution on $[b, c], b<c<$ $\eta(b)\}$, and let $S^{*}$ be the unique solution of (MR) on $\left[b, c_{1}\right)$. Since the derivative of every nontrivial vector solution $y$ of $(\mathrm{E})$ with $y(b)=0$ does not vanish on $[b, \eta(b))$, it follows from the necessity of Theorem 1 that (MR) has a solution, say $S^{\circ}$, on $[b, \eta(b))$; thus, $S^{*}=S^{\circ}$ on $\left[b, c_{1}\right)$ by the uniqueness of solutions. If $c_{1}<\eta(b)$, we may assume that $S^{*}$ is defined on $\left[b, c_{1}\right]$ (by setting $S^{*}\left(c_{1}\right)=\lim _{x \rightarrow c_{1}^{-}} S^{*}(x)=$ $S^{\circ}\left(c_{1}\right)$, if necessary). The solution $S^{*}$ may then be continued to a right neighborhood $\left[c_{1}, c_{1}+\varepsilon\right], \varepsilon>0$, of $c_{1}$ [7, p. 15]. This implies that (MR) has a unique solution on $\left[b, c_{1}+\varepsilon\right], \varepsilon>0$, contrary to the choice of $c_{1}$. Therefore, $c_{1}=\eta(b)$ and (MR) has a unique solution $S$ on $J$.

The solution $S$ is continuously differentiable because $R^{-1}$ and $P$ are continuous. Furthermore, $S$ is nontrivial because $R^{-1} \not \equiv 0-R^{-1}$ is invertible on $[b, \eta(b))$ and it cannot have zero rows or zero columns at any point of $[b, \eta(b))$ - and it may be obtained as the uniform limit of the successive approximations $\left\{S_{i}\right\}$ defined recursively by the formula

$$
\begin{gathered}
S_{0}(x)=0, \\
S_{k+1}(x)=\int_{b}^{x} R^{-1}(t) d t+\int_{b}^{x} S_{k}(t) P(t) S_{k}(t) d t,
\end{gathered}
$$

$k=0,1, \ldots$, on some interval [ $b, d], b<d<\eta(b)$ (see, e.g., [7, p. 12]). Due to the inequalities (1), $S_{k} \geqslant 0$ on $[b, d], k=0,1, \ldots$, and therefore the uniform limit $S \geqslant 0$ on $[b, d]$. Let $d_{1}=\sup \{d: S \geqslant 0$ on $[b, d], b<d<\eta(b)\}$. Then $S \geqslant 0$ on $\left[b, d_{1}\right)$. We shall prove that $d_{1}=\eta(b)$. If $d_{1}<\eta(b)$, then $0 \leqslant S<\infty$ on $\left[b, d_{1}\right]$ by the continuity of $S$. In this case, $S$ may again be represented on some interval $\left[d_{1}, e\right], d_{1}<e<\eta(b)$, as the uniform limit of the successive approximations

$$
\begin{gathered}
S_{0}(x)=S\left(d_{1}\right) \geqslant 0, \\
S_{k+1}(x)=S\left(d_{1}\right)+\int_{d_{1}}^{x} R^{-1}(t) d t+\int_{d_{1}}^{x} S_{k}(t) P(t) S_{k}(t) d t,
\end{gathered}
$$

$k=0,1, \ldots$ Since $S_{k} \geqslant 0$ on $\left[d_{1}, e\right], k=0,1, \ldots, S \geqslant 0$ on $\left[d_{1}, e\right]$. We are thus led to the conclusion that $S \geqslant 0$ on $[b, e]$, contrary to the choice of $d_{1}$. Consequently, $d_{1}=\eta(b)$ and $S \geqslant 0$ on $[b, \eta(b))$.

Returning now to the proof of Theorem 1, we shall first prove that $\left|Y^{\prime}\right|$, the determinant of $Y^{\prime}$, does not vanish on $[b, \omega)$ if (MR) has a solution $S$ on $[b, \omega)$. Since $\left|Y^{\prime}\right|$ is continuous and $\left|Y^{\prime}(b)\right|=1$ by $(\mathrm{M}),\left|Y^{\prime}\right|$ does not vanish on some right neighborhood $N$ of the point $b$, that is, $Y^{\prime}$ is invertible on $N$. Since $R$ is invertible, $Y\left(R Y^{\prime}\right)^{-1}$ is defined on $N$ and satisfies (MR), as was shown earlier. Due to the uniqueness of solutions of the initial value problem (MR) proved in Lemma 1, we 
have $S=Y\left(R Y^{\prime}\right)^{-1}$ on $N$. Suppose that $\left|Y^{\prime}\right|$ vanishes at some point on $[b, \omega)$ : Let $\tilde{x}$ be the first point to the right of $b$ at which $\left|Y^{\prime}\right|$ vanishes. Then there exists a nonzero constant vector $\beta$ such that $Y^{\prime}(\tilde{x}) \beta=0$. On the interval $[b, \tilde{x})$ we have $S=$ $Y\left(R Y^{\prime}\right)^{-1}$, which may be written as $S R Y^{\prime}=Y$; this equality is indeed valid on $[b, \tilde{x}]$ because $S, R, Y$ and $Y^{\prime}$ are continuous on $[b, \tilde{x}]$. In particular, $S(\tilde{x}) R(\tilde{x}) Y^{\prime}(\tilde{x}) \beta=Y(\tilde{x}) \beta=0$. But this is absurd since $w \equiv Y \beta$ is a nontrivial solution of $(\mathrm{E})$ and it cannot satisfy the condition $w(\tilde{x})=w^{\prime}(\tilde{x})=0$. Therefore, $\left|Y^{\prime}\right|$ cannot vanish on $[b, \omega)$.

If $y$ is any nontrivial solution of (E) with $y(b)=0$, then there exists a nonzereo constant vector $\gamma$ such that $y=Y \gamma$. Evidently, $y^{\prime}=Y^{\prime} \gamma \neq 0$ on $[b, \omega)$ because $\left|Y^{\prime}\right| \neq 0$ on $[b, \omega)$. This completes the proof.

Another result we need for proving comparision theorems is a version of Lemma 3.2 [12], strengthened for the matrix Riccati systems

$$
S^{\prime}=R_{i}^{-1}+S P_{i} S, \quad S(b)=0, \quad i=1,2 .
$$

LeMma 2. Let $R_{i}$ and $P_{i}$ be $n \times n$ matrices with continuous and real elements and let $R_{i}$ be invertible on an interval $[a, \omega), i=1,2$. Assume that

$$
0 \leqslant \int_{b}^{x} R_{2}^{-1}(t) d t \leqslant \int_{b}^{x} R_{1}^{-1}(t) d t, \quad 0 \leqslant P_{2}(x) \leqslant P_{1}(x), \quad b \leqslant x<\omega,
$$

for some $b, a \leqslant b<\omega$. If there exists a nonnegative differentiable matrix $S$ defined on $[b, \omega)$ satisfying the matrix inequality

$$
S^{\prime} \geqslant R_{1}^{-1}+S P_{1} S, \quad S(b)=S_{b} \geqslant 0,
$$

then the matrix differential equation

$$
T^{\prime}=R_{2}^{-1}+T P_{2} T, \quad T(b)=T_{b}, \quad S_{b} \geqslant T_{b} \geqslant 0,
$$

has a continuous solution $T \leqslant S$ on $[b, \omega)$.

Proof. The existence of $T$ is proved by the iteration procedure

$$
T_{0}(x)=S, \quad T_{k+1}(x)=T_{b}+\int_{b}^{x} R_{2}^{-1}(t) d t+\int_{b}^{x} T_{k}(t) P_{2}(t) T_{k}(t) d t,
$$

$b \leqslant x \leqslant \omega, k=0,1, \ldots$ (cf. [12]). For $k=0$,

$$
\begin{aligned}
0 & \leqslant T_{1}(x)=T_{b}+\int_{b}^{x} R_{2}^{-1}(t) d t+\int_{b}^{x} S(t) P_{2}(t) S(t) d t \\
& \leqslant S_{b}+\int_{b}^{x} R_{1}^{-1}(t) d t+\int_{b}^{x} S(t) P_{1}(t) S(t) d t \leqslant S(x)=T_{0}(x),
\end{aligned}
$$

due to (3), (4), (5) and the nonnegativity of $S$; hence, $T_{1}$ is continuously differentiable and $0 \leqslant T_{1} \leqslant T_{0}$ on $[b, \omega)$. From (6) we see that $T_{k+1} \geqslant 0$ if $T_{k} \geqslant 0$. Also, for $k=0,1, \ldots$,

$$
T_{k+1}(x)-T_{k}(x)=\int_{b}^{x}\left[T_{k}(t) P_{2}(t) T_{k}(t)-T_{k-1}(t) P_{2}(t) T_{k-1}(t)\right] d t
$$

where the integrand is nonpositive if $0 \leqslant T_{k} \leqslant T_{k-1}$. Therefore, $0 \leqslant T_{k+1} \leqslant T_{k}$ if $0 \leqslant T_{k} \leqslant T_{k-1}$. Since $0 \leqslant T_{1} \leqslant T_{0}$, the sequence of continuously differentiable matrices $\left\{T_{k}\right\}$ decreases monotonically and is bounded below by zero. Furthermore, 
the sequence is equicontinuous on any compact subinterval $K$ of $[b, \omega)$. To show this, let $\|A\|$ be the norm of an $n \times n$ matrix $A=\left(a_{i j}\right)$ defined by $\|A\|=\sum_{i, j=1}^{n}\left|a_{i j}\right|$. Let $M>0$ be a constant such that $\left\|R_{2}^{-1}\right\|,\left\|P_{2}\right\|$, and $\left\|T_{k}\right\|, k=0,1, \ldots$, are all bounded by $M$ on $K$. From (6),

$$
T_{k+1}\left(x_{2}\right)-T_{k+1}\left(x_{1}\right)=\int_{x_{1}}^{x_{2}} R_{2}^{-1}(t) d t+\int_{x_{1}}^{x_{2}} T_{k}(t) P_{2}(t) T_{k}(t) d t,
$$

$x_{1}, x_{2} \in K, k=0,1, \ldots$ Thus,

$$
\begin{aligned}
\left\|T_{k+1}\left(x_{2}\right)-T_{k+1}\left(x_{1}\right)\right\| & \leqslant \int_{x_{1}}^{x_{2}}\left\|R^{-1}(t)\right\||d t|+\int_{x_{1}}^{x_{2}}\left\|T_{k}(t) P_{2}(t) T_{k}(t)\right\||d t| \\
& \leqslant\left(M+M^{3}\right)\left|x_{2}-x_{1}\right|, \quad x_{1}, x_{2} \in K,
\end{aligned}
$$

$k=0,1, \ldots$, and this implies that the sequence $\left\{T_{k}\right\}$ is equicontinuous on $K$. Since it is also uniformly bounded on $K,\left\{T_{k}\right\}$ converges uniformly on $K$. The uniform limit $T=\lim _{k \rightarrow \infty} T_{k}$ is a continuous solution of (5) and $T \leqslant T_{0}=S$ on $K$. Since this conclusion holds for every compact subinterval of $[b, \omega)$, it holds for $[b, \omega)$.

We are now ready to prove a comparison theorem for $\eta_{i}(x)$, the right-hand pseudoconjugate function of $\left(\mathrm{E}_{i}\right), i=1,2$, defined on $[a, \omega)$.

THEOREM 2. Let $b$ be a point on the interval $[a, \omega)$. If

$$
R_{1}^{-1}(x) \geqslant 0, \quad \int_{b}^{x} R_{1}^{-1}(t) d t \geqslant \int_{b}^{x} R_{2}^{-1}(t) d t \geqslant 0, \quad P_{1}(x) \geqslant P_{2}(x) \geqslant 0,
$$

$b \leqslant x<\omega$, then $\eta_{1}(b) \leqslant \eta_{2}(b)$. If the stronger condition

$$
R_{1}^{-1}(x) \geqslant R_{2}^{-1}(x) \geqslant 0, \quad P_{1}(x) \geqslant P_{2}(x) \geqslant 0,
$$

$a \leqslant x<\omega$, holds, then $\eta_{1}(b) \leqslant \eta_{2}(c), a \leqslant b \leqslant c<\omega$.

Proof. Every nontrivial solution $y$ of $\left(\mathrm{E}_{1}\right)$ with $y(b)=0$ has the property that $y^{\prime} \neq 0$ on $\left[b, \eta_{1}(b)\right)$. Hence, the corresponding matrix Riccati equation $\left(\mathrm{MR}_{1}\right)$ has a solution $S$ on $\left[b, \eta_{1}(b)\right)$ by Theorem 1 . The solution $S$ is nontrivial and nonnegative on $\left[b, \eta_{1}(b)\right)$ by Lemma 1 . According to (7) and Lemma 2, the matrix Riccati system $\left(\mathrm{MR}_{2}\right)$ associated with $\left(\mathrm{E}_{2}\right)$ has a continuous solution $T$ on $\left[b, \eta_{1}(b)\right)$. Therefore, by Theorem 1 , every nontrivial solution vector $w$ of $\left(\mathrm{E}_{2}\right)$ with $w(b)=0$ has the property that $w^{\prime} \neq 0$ on $\left[b, \eta_{1}(b)\right)$; consequently, $\eta_{1}(b) \leqslant \eta_{2}(b)$.

If (8) holds and $c$ is an arbitrary point of $[a, \omega)$, then

$$
\int_{c}^{x} R_{1}^{-1}(t) d t \geqslant \int_{c}^{x} R_{2}^{-1}(t) d t \geqslant 0, \quad a \leqslant c \leqslant x<\omega .
$$

For $a \leqslant b \leqslant c<\eta_{1}(b)$, $\left(\mathrm{MR}_{1}\right)$ has a nontrivial solution $S$ which is continuous and nonnegative on $\left[b, \eta_{1}(b)\right)$ by Theorem 1 and Lemma 1 . Applying Lemma 2 to the interval $\left[c, \eta_{1}(b)\right)$, we conclude that the system $T^{\prime}=R_{2}^{-1}+T P_{2} T, T(c)=0$, has a matrix solution $T$ on $\left[c, \eta_{1}(b)\right)$. Again by Theorem 1 , if $v$ is any nontrivial solution of $\left(\mathrm{E}_{2}\right)$ with $v(c)=0$, then $v^{\prime}$ does not vanish on $\left[c, \eta_{1}(b)\right)$. Therefore, $\eta_{1}(b) \leqslant \eta_{2}(c)$, $a \leqslant b \leqslant c<\eta_{1}(b)$.

If, on the other hand, $\eta_{1}(b) \leqslant c<\omega$, it is obvious that $\eta_{1}(b) \leqslant \eta_{2}(c)$. This completes the proof. 
When we put $R_{1}=R_{2}=R$ and $P_{1}=P_{2}=P$ in Theorem 2-many comparison theorems for the second-order systems $\left(\mathrm{E}_{i}\right), i=1,2$, fail to hold for this case-we obtain the following "separation theorem": If $R$ is invertible, $R^{-1} \geqslant 0$, and $P \geqslant 0$ on $[a, \omega)$, then the equation (E) has no nontrivial solution $y$ such that $y\left(x_{1}\right)=$ $y^{\prime}\left(x_{2}\right)=0, b \leqslant x_{1} \leqslant x_{2}<\eta(b)$, for any $b, a \leqslant b<\omega$. This result is equivalent to the statement that $\eta(x)$ is a nondecreasing function of $x$ on $[a, \omega)$.

Let $\phi_{i}(x)$ be the right-hand focal point of $x$ for the equation $\left(\mathrm{E}_{i}\right), i=1,2$. There are analogous comparison results for $\phi_{i}(x), i=1,2$, which we summarize below.

Let $U$ be the solution of the matrix system

$$
\left(R(x) U^{\prime}\right)^{\prime}+P(x) U=0, \quad U(b)=I, \quad U^{\prime}(b)=0,
$$

for some $b, a \leqslant b<\omega$. Put $V=-R U^{\prime} U^{-1}$. If every nontrivial solution $y$ of (E) with $y^{\prime}(b)=0$ does not vanish on $[b, \omega)$, then $U$ is invertible on $[b, \omega)$. Thus, $V$ is defined on $[b, \omega)$ and satisfies thereon

$$
V^{\prime}=P+V R^{-1} V, \quad V(b)=0 .
$$

This proves the necessicy part of the following theorem.

THEOREM 3. Suppose that $R$ and $P$ are $n \times n$ matrices with continuous and real elements and that $R$ is invertible on an interval $[a, \omega)$. Let $b$ be a point on $[a, \omega)$. Every nontrivial solution vector $y$ of $(\mathrm{E})$ with $y^{\prime}(b)=0$ does not vanish on $[b, \omega)$ if and only if the matrix Riccati system $\left(\mathrm{MR}^{\prime}\right)$ has a solution on $[b, \omega)$.

Sufficiency of this theorem may be proved in a manner similar to the corresponding proof of Theorem 1, using the following analogue of Lemma 1.

LEMMA 3. The matrix Riccati equation ( $\left.\mathrm{MR}^{\prime}\right)$ has a unique solution on $[b, \phi(b))$, which is continuously differentiable. The solution is nontrivial if $P \neq 0$ and it is nonnegative on $[b, \phi(b))$ if $R^{-1}(x) \geqslant 0, P(x) \geqslant 0, b \leqslant x<\phi(b)$.

Using Theorem 3, Lemma 2 (with $P_{i}$ and $R_{i}^{-1}$ interchanged in (3), (4) and (5), $i=1,2)$ and Lemma 3 , we can similarly prove the following comparison theorem for $\phi_{i}(x)$.

THEOREM 4. If, for some $b, a \leqslant b<\omega$,

$$
P_{1}(x) \geqslant 0, \quad \int_{b}^{x} P_{1}(t) d t \geqslant \int_{h}^{x} P_{2}(t) d t \geqslant 0, \quad R_{1}^{-1}(x) \geqslant R_{2}^{-1}(x) \geqslant 0,
$$

$b \leqslant x<\omega$, then $\phi_{2}(b) \geqslant \phi_{1}(b)$. Moreover, if

$$
P_{1}(x) \geqslant P_{2}(x) \geqslant 0, \quad R_{1}^{-1}(x) \geqslant R_{2}^{-1}(x) \geqslant 0,
$$

$a \leqslant x<\omega$, then $\phi_{2}(c) \geqslant \phi_{1}(b), a \leqslant b \leqslant c<\omega$.

Putting $P_{1}=P_{2}=P$ and $R_{1}=R_{2}=R$ in Theorem 4, we again obtain a "separation theorem", which is equivalent to the statement that $\phi(x)$ is a nondecreasing function of $x$. 


\section{REFERENCES}

1. S. Ahmad and A. C. Lazer, On the components of extremal solutions of second order systems, SIAM J. Math. Anal. 8 (1977), 16-23.

2. An N-dimensional extension of the Sturm separation and comparison theory to a class of nonselfadjoint systems, SIAM J. Math. Anal. 9 (1978), 1137-1150.

3. On an extension of Sturm's comparison theorem to a class of nonselfadjoint second-order sistems, Nonlinear Anal. 4 (1980), 497-501.

4. G. Birkhoff and G. C. Rota, Ordinary differential equations, 3rd ed., Wiley, New York, 1978.

5. G. J. Butler and L. H. Erbe, Nonlinear integral Riccati systems and comparison theorems for linear differential equations, SIAM J. Math. Anal. 14 (1983), 463-473.

6. S. Cheng, Nonoscillatory solutions of $x^{(m)}=(-1)^{m} Q(t) x$, Canad. Math. Bull. 22 (1979), 17-21.

7. E. A. Coddington and N. Levinson, Theory of ordinary differential equations, McGraw-Hill, New York, 1955.

8. E. Hille, Non-oscillation theorems, Trans. Amer. Math. Soc. 64 (1948), 234-252.

9. W. J. Kim, Generalized comparison theorems for disfocality types of the equation $L_{n} . y+p y=0, \mathrm{~J}$. Math. Anal. Appl. 109 (1985), 182-193.

10. M. K. Kwong, Matrix Riccati inequality and oscillation of second order differential systems, Lecture Notes in Math., vol. 1032, Springer-Verlag, New York, 1982.

11. M. Morse, A generalization of the Sturm separation and comparision theorems in $n$-space, Math. Ann. 103 (1930), 52-69.

12. Z. Nehari, Nonlinear techniques for linear oscillation problems, Trans. Amer. Math. Soc. 210 (1975), 387-406.

13. W. T. Reid, Sturmian theory for ordinary differential equations, Appl. Math. Sci., vol. 31, SpringerVerlag, New York, 1980.

14. H. L. Smith, A note on disconjugacy for second order systems, Pacific J. Math. 89 (1980), 447-452.

15. E. C. Tomastik, Comparison theorems for second order nonselfadjoint differential systems, SIAM J. Math. Anal. 14 (1983), 60-65.

Department of applied Mathematics and Statistics, State University of New York, Stony BROOK, NEW YORK 11794 